\title{
The Human Microbiome, Conventional Medicine, and Homeopathy
}

\author{
Ronald D. Whitmont ${ }^{1}$ \\ ${ }^{1}$ Department of Family and Community Medicine, New York Medical \\ College, Rhinebeck, New York, United States \\ Homeopathy 2020;109:248-255.
}

Address for correspondence Ronald D. Whitmont, MD, Department of Family and Community Medicine, New York Medical College, 6250 Route 9, Rhinebeck, New York 12572, United States (e-mail: homeopathicmd@gmail.com).
Abstract
Keywords
- microbiome
- health ecology
- homeopathy
- symbiosis
- dysbiosis
- holism
- psychosomatics
- direction of cure
- Law of Similars
- individuality and susceptibility
- minimum dose
- homeostasis

Human health is intimately linked to the ecology and diversity of the human microbiome. Together, the human organism and the human microbiome work as a complex super-organism throughout the human life cycle. Microbiome science provides direct evidence and substantiation of the fundamental principles of homeopathy, including holism, psychosomatics, direction of cure, the Law of Similars, individuality and susceptibility, minimum dose, and homeostasis. Whilst many conventional (allopathic) medical treatments irreversibly damage the ecology of the microbiome and trigger chronic immune dysfunction and inflammation, the future sustainability of the entire field of medicine depends on the ability to recognize these inconvenient biological truths and to embrace a safer approach based on this evidence. Fortunately, one of the oldest forms of clinically verifiable, evidence-based, and ecologically sustainable medicine, that does not harm the microbiome, already exists in the form of homeopathy.

\section{Introduction}

After completion of the Human Genome Project, the National Institutes of Health turned the tools of genetic sequencing toward the study of the entire library of microbiologic life in and on the human body. These investigations, carried out worldwide, have determined that there are more than ten thousand different microbial species and more than one hundred trillion different individual organisms colonizing the average normal healthy human body. The actual quantity of individual "non-human" micro-organisms in the human microbiome outnumbers human cells; ${ }^{1}$ contains more genetic information than human DNA; $;^{2}$ weighs nearly $1.5 \mathrm{~kg}$; forms an invisible "organ" or continuous living bio-shield (biofilm) of non-human tissue that covers every square millimeter of skin; lines every orifice (including the respiratory, gastrointestinal [GI], and urogenital tracts); exists within every internal organ, including the brain, ${ }^{3}$ liver, ${ }^{4}$ placenta, ${ }^{5}$ uterus, ${ }^{6}$ and blood $;{ }^{7}$ and functions as an indepen-

received

December 17, 2019

accepted after revision

February 17, 2020

published online

May 14, 2020

dent, inter-connected, essential "organ" throughout the human body.

The microbiome is composed of a vast complicated network of micro-organisms that criss-cross, bridge, and inter-connect, communicating (with each other and with us) via chemical, structural, electrical, and quorum-sensing throughout the entire body. ${ }^{8}$ No healthy organ system exists without a microbiome. Network connections between organisms are capable of utilizing the equivalent of a type of biological "internet", providing parallel systems of communication, circulation, nutrient exchange, immunological, and structural support throughout the tissues and glands of the human body. ${ }^{9}$

This diverse, ubiquitous amalgamation of human tissue with non-human micro-organisms interacts and cooperates in every essential aspect of life, beginning with sexual attraction, bonding, reproduction and conception onwards, supporting the development of the entire body including the nervous, immune, digestive, respiratory, and sexual reproductive systems. ${ }^{10}$ The microbiome-human relationship is a complex,

Copyright @ 2020 The Faculty of Homeopathy
DOI https://doi.org/ 10.1055/s-0040-1709665. ISSN $1475-4916$. 
fundamental, synergistic symbiosis that functions as a "superorganism" of integrated and mutually inter-dependent species. ${ }^{11}$ Not only does human life depend on the microbiome for normal healthy growth, development and survival, but the microbiome, which has adapted itself to the human organism, is reciprocally dependent on the human body for its own survival. $^{12}$

The microbiome is not simply an opportunistic superinfection representing a casual encounter with micro-organisms, but one of the most fundamental symbioses of nature, that has likely enabled the process of species evolution itself to take place. The microbiome has provided the basis for the rapid development and fine-tuning of events such as consciousness, intellect and intelligence, personality and mood. ${ }^{13}$ Yet modern technological societies now stand on the brink of self-destruction, the result of biologically destructive forces that were unleashed in the form of modern allopathic medical treatment, which threaten to destroy the microbiome and create an immunologic "tsunami" risking human health and species survival.

The discovery of the human microbiome represents "cutting edge" science, and one of the most important milestones of modern medicine. Microbiome science reveals how longstanding assumptions and practices, firmly established over many decades as "standards of care" in allopathic medicine, are simply unsustainably destructive and irreversibly harmful to human, microbiome, and environmental health. Evidence-based insight garnered from microbiome science challenges and provides qualitative proof that the most fundamental foundations of modern allopathic medicine, particularly man's adversarial relationship with the environment and other species in it, are destroying the microbiome and promoting a worldwide epidemic of chronic inflammatory illness. ${ }^{14}$ The "inconvenient truth" is that modern medicine, a "wunderkind" of science and technology, has engineered a vast epidemic of human and environmental illness by systematically and unwittingly destroying the microbiome and the environment.

Ironically, the youngest branch of medical research, using the world's most advanced and sophisticated tools of genetic sequencing, is providing evidence verifying the fundamental principles and premises of homeopathy (one of the most controversial and intensely debated fields of medicine). Most of the science emerging from the study of the microbiome is fundamentally consistent with the principles and practices of homeopathic medicine, which has already been in worldwide use for more than two centuries. Homeopathy may prove to be one of the most scientifically justified, safest, and promising methods of supporting long-term health because it promotes both microbiome and environmental health and sustainability. True science (not just economically driven innovation) demands a fundamental paradigm-shifting realignment of medicine to work within an ecologically sustainable framework of health promotion. To achieve this, the fields of both allopathic and homeopathic medicine must be re-examined in light of microbiome science to determine the course of future sustainable, wellness-promoting healthcare.
What follows is a summary of some of the most pertinent discoveries made in microbiome science that relate to the current thesis.

\section{The Human Microbiome and Homeopathy}

The human microbiome has been described as an "invisible biological organ", which plays a critical role in metabolism, development and homeostasis, and directly modulates the host inflammatory immune response. ${ }^{15}$ It is a "super-organ" that controls many functions that are equivalent to, and in some cases more important than, the solid organs of the human host. ${ }^{16}$ The microbiome regulates digestion and absorption of nutrients, along with growth and development of the immune and nervous systems. It directly contributes to behavior and mood, social interaction, personality development, sexual development, sexual attraction, reproduction, and fertility. ${ }^{10}$ The microbiome influences most (if not all) metabolic functions, including energy production, body temperature, and tissue growth. ${ }^{17}$ It regulates the development and function of both the innate and adaptive immune systems, ${ }^{18}$ prevents diseases of immune dysfunction, ${ }^{19}$ neutralizes enteric pathogens, metabolizes and neutralizes dietary carcinogens, and favorably modulates the host's immune response to resist infection and disease. ${ }^{20}$ The microbiome aids in complex carbohydrate fermentation and absorption, provides a significant source of a range of essential vitamins (particularly those of the B and K groups), and keeps pathogenic micro-organisms in check. The GI tract microbiome aids in the development of GI barrier function, integrity, and systemic immune responses, while promoting formation of a tolerant state between gut organisms and the immune system, which regulates tight junction structure and function. ${ }^{21}$

More than 200 years ago, without any knowledge of microbiology or genetics, the physician-scholar, and founder of homeopathy, Christian Friedrich Samuel Hahnemann, wrote:

"In the healthy human state, the spirit-like [invisible] life force (autocracy) that enlivens the material organism as dynamis, governs without restriction and keeps all parts of the organism in admirable, harmonious, vital operation, as regards both feelings and functions, so that our indwelling, rational spirit can freely avail itself of this living, healthy instrument for the higher purposes of our existence." $22^{22}$

More than two centuries before its discovery and without technical knowledge of its existence, Hahnemann qualitatively described the role that the human microbiome plays in health. He attributed this phenomenon to non-physical, spiritual forces, because it was not until microbiome scientists discovered that this "invisible organ" was made up of an extensive network of bacteria, viruses, fungi, archaea, and protists interacting directly with the human organism that his observations could be attributed to the microbiome. Hahnemann described the qualitative behavior of the human microbiome long before it was detected microscopically, 
classified, and studied with the most modern tools of molecular biology and computer processing.

Microbiome science was actually pioneered in the early 20th century by the British homeopathic physicians John Paterson and Edward Bach, who studied the changes in the bowel flora associated with a wide range of systemic illnesses. Between 1927 and 1932, Paterson cultured more than 8,000 stool specimens and published results, finding that "Homeopathic potencies are capable of completely altering the bacterial flora of the bowel, and this fact has been demonstrated in many hundreds of cases". ${ }^{23} \mathrm{He}$ noted that the gut flora changed along with different diseases and that it was altered favorably with homeopathic curative intervention. These physicians also pioneered the use of a group of specific homeopathic medicines prepared directly from microbiologic isolates of disturbed (dysbiotic) stool from diseased individuals. This group of medicines, collectively known as the "bowel nosodes", proved to be effective as interventions, that continue to be used today to treat a wide range of disturbances both within and outside of the GI tract. In recent years, the bowel nosodes have demonstrated particular benefit in re-establishing functional recovery in many individuals suffering from iatrogenicinduced dysbioses. In some respects, these homeopathic physicians pioneered the first study of fecal microbiome transplants by demonstrating the clinical benefits of using stool-derived isolates to re-establish health and resolve inflammation. This early research provided clinical evidence of homeopathy's benefit in addressing dysbiosis long before it had even been named. Modern clinical research confirms that homeopathic medicines (not just bowel nosodes) directly alter many cellular functions ranging from messenger RNA expression to protein synthesis. ${ }^{24}$ It is entirely tenable, based on both pre-clinical and clinical evidence, that homeopathic medicines act directly on the microbiome, both on individual organisms and on the collective super-organism, and that health and immune system benefits arise directly via this route.

The behavior of the microbiome coincides closely with what Hahnemann qualitatively described as a non-physical (spiritual, invisible) entity, or "vital force" that interacts with the human organism and continually guides it towards health. Hahnemann only had archaic language, unsophisticated in the fields of biochemistry, microbiology, and genetics (because these disciplines did not exist) to describe these clinical phenomena. It is more than likely that what Hahnemann qualitatively described as a "vital force" is precisely what molecular biologists have collectively called the microbiome, since these two entities act identically in living systems. The entire field of homeopathy is based on observations made describing the characteristics of this entity, and if confirmed, homeopathy may turn out to be one of the earliest forms of sustainably managing the human microbiome. Hahnemann may have been the first to describe one of the most important, gentle, effective, and sustainable methods of manipulating the microbiome, foreshadowing its discovery by more than two centuries.

The most important and fundamental principles of homeopathy can be verified by evidence revealed by microbiome science, reflected in the universal health principles of:
- Holism

- Psychosomatics

- Direction of cure

- Law of Similars

- Individuality and susceptibility

- Minimum dose.

Each of these is briefly outlined below.

\section{Holism}

"As above, so below, as within, so without, as the universe, so the soul..." - Hermes Trismegistus

Holism describes the phenomenon that every intervention (local or systemic) ultimately affects the entire body and all its parts, no matter if this was the desired or intended effect, or not. Results that are sometimes referred to as "side effects" (from allopathic medicines) are simply the predictable systemic effects of locally applied treatments acting on the rest of the body. Conventional medical practices assert that single parts of the body are somehow distinct and separate entities that can be studied and treated in isolation, without affecting the entire organism (in some way). This mistaken approach stems from a combination of a 16th century Cartesian philosophical exercise, separating the mind from the body (that has been repeatedly demonstrated to be false) and convenience. Organ systems are theoretically categorized as separate and distinct entities, but these are artificial distinctions that simplify the study and treatment, making it easier and more convenient for the practitioner. In actuality, every single organ system of the body interacts and is connected structurally, biochemically, hormonally, electrically, and through the microbiome, ${ }^{8}$ with every other organ. Treating conditions and organs without regard for their holistic interconnection ultimately imperils the health of the entire organism, promotes polypharmacy, drug overuse, and contributes to the epidemics of addiction, ${ }^{25}$ antibiotic resistance, ${ }^{26}$ and a wide array of other adverse outcomes that increase the risk and cost of conventional medical care.

Even a rudimentary study of homeopathy demonstrates the benefit of prescriptions that are based on the holistic "totality of symptoms" of the entire organism. Prescribing from this premise increases global therapeutic efficacy and long-term benefit. Homeopathic treatments are, by definition, based on addressing the totality of all the symptoms expressed by an individual. Even local prescribing, using homeopathic medicines, tends generally to improve the health of the entire organism rather than complicating it with untoward side effects.

"Homeopathic symptoms are manifestations of a singular, pervasive, emergent disturbance of the state of the individual as a whole system or network of networks, not of a specific body part or biological mechanism in isolation." 27 
One of the plagues of allopathic medicine is the epidemic of adverse effects, which represent the unintended side effects of treatments that were selected on the basis of local organ system management without anticipating or considering the systemic (holistic) effects on the health of the entire organism. ${ }^{28}$

\section{Psychosomatics}

Psychosomatics is an important corollary of the principle of holism that confirms the relationship between physical and mental/emotional functioning. Mental/emotional and physical symptoms appear to be part of a continuum of health and illness that are almost always inter-connected. Most diseases have both mental and physical correlates. Nearly every single medicine (homeopathic and allopathic) affects both mental and physical functioning, ${ }^{29}$ depending on dosage, route of administration and duration of use. Homeopathic science includes the study of these effects and takes advantage of their connection in the therapeutic intervention process.

By considering both mental and physical symptoms together, as part of the same holistic totality, homeopathic treatment simultaneously addresses and impacts both, by design. Utilizing a psychosomatic perspective, as part of the process of case analysis and treatment, increases the therapeutic efficacy, global response rate, and quality of outcome.

One of the key discoveries of microbiome science is the connection between the gut microbiome and cognitiveemotional-mental and physical functioning. Local changes in the GI microbiome profoundly affect the growth, development and health of the entire organism, and vice versa. Not only does the state of the GI microbiome impact the brain, its moods and cognitive abilities, ${ }^{30}$ but these moods, emotions and mental attitudes also impact the gut microbiome and alter microbial balance and diversity in the GI tract in the reverse direction. ${ }^{31}$ The microbiome, which exists within the healthy brain, is integral to the development and maintenance of consciousness, memory, emotion and cognition. ${ }^{32}$ The ecology of micro-organisms living within the brain is critical to the nervous system function, and plays a role in the definition of "self", influencing the risk of developing mental, emotional and mood disorders. ${ }^{33}$ Animals raised in germfree conditions display significant impairment in neurologic and social development, while in the wild it is the dominant male chimpanzees that are the most heavily parasite-ridden and microbiome-dense individuals of the tribe. ${ }^{34}$

\section{Direction of Cure}

Homeopaths have long-observed clinically that when health deteriorates, illness and inflammation proceed along certain well-defined gradient planes and pathways: from acute to chronic illness; from more superficial structures to deeper and more vital tissues, etc. Corresponding to these patterns of illness progression, there are mirror-image patterns that correspond to health-sustaining recovery. As recovery from a serious illness takes place, symptoms frequently progress through systems in the reverse order of appearance. As recov- ery proceeds, illness leaves deeper structures and vital organs and moves to more superficial structures and less vital organs; recovery proceeds in a cephalo-caudal, or dorsal-ventral pattern; centrifugally, and along gradient planes corresponding to embryological stages of development. These tendencies were well described by the homeopathic physician and educator, Constantine Hering, ${ }^{35}$ and others. ${ }^{36}$

Homeopathic therapy frequently demonstrates the curious phenomenon of "direction of cure", whereby as chronic illness improves or resolves, acute illnesses tend to recur: "If any superficial inflammatory disease was suppressed, at this point it returns, and such a return is a further confirmation of the immune system reverting to its previous state". ${ }^{37}$ This phenomenon is more likely to be noticed if the holistic and psychosomatic totality of the entire body is considered in the treatment and evaluation process.

Microbiome science confirms that when acute illnesses are treated by methods that damage the microbiome and/or inhibit the immune system, then acute illnesses frequently appear temporarily to abate, and are later replaced by more severe chronic states of inflammation. Routine and frequent use of antibiotics in early childhood is directly linked with an increased risk of developing chronic inflammation in the form of allergies, asthma, and autoimmune disease later in childhood. ${ }^{38-42}$ Acute illness, treated without concern for the ecology of the microbiome or the integrity of the immune system, frequently progresses in this manner toward more serious pathology and chronic inflammatory disorders. The pediatric population suffers most heavily from these microbiologically destructive events, with skyrocketing rates of chronic systemic inflammatory illness. ${ }^{43}$

"When continuously thwarted through anti-inflammatory drugs or other substances that hinder inflammation, [the body] loses the ability to react through strong inflammation and enters a state of chronic low-grade inflammation, eventually turning on the chronic disease that one is predisposed to." 44

\section{Law of Similars}

"Every action produces an equal and opposite reaction" - Sir Isaac Newton, $3^{\text {rd }}$ Law of Motion

The Law of Similars is a fundamental principle of homeopathy that describes the phenomenon that medicines demonstrate greatest effectiveness when selected on the basis of their (previously demonstrated/proven) capacity to evoke a similar state of pathology most closely matching the state of a person who is sick and being treated. The degree of similarity between the medicine's pathogenicity and the patient's actual disturbance is directly proportional to the likelihood that the body will respond to treatment.

The therapeutic response pattern produced by a similar pathogenic medicine results not from the drug's pathogenic chemical/primary action, but from the body's secondary biologically adaptive reaction to the drug. 
"The body as a complex adaptive system has the capacity for functional self-reorganization, emergence, and multiscale self-similarity over global and local levels of organization." 27

This secondary adaptive response is always opposite to the drug's primary chemical effects (i.e., the body's secondary response to the primary pain-relieving effect of opiates is an upregulation of pain receptors, increased pain sensitivity, and hyperesthesia). Similarly, by down-regulating inflammatory pathways in a pulsatile fashion, chronic use of nonsteroidal anti-inflammatory drugs promotes compensatory up-regulation of these same pathways and shifts the host baseline equilibrium toward an inflammatory state. ${ }^{45}$ Homeopathic prescribing is based on both these phenomena: selecting a drug that is most similar to the actual pathology, and utilizing the body's secondary adaptive response tendency. The use of similars may appear superficially paradoxical (from a chemical perspective), but it is intuitive from a biologic perspective, since it recruits the body's adaptive network response (homeostasis), integral to all living systems. ${ }^{46}$ Secondary adaptive reactions tend to be more sustained than the primary chemical drug effects since they are part of the body's adaptive response system rather than being dependent on the half-lives of chemical agents. ${ }^{27}$ Homeopathy is unique in recruiting this paradoxical secondary adaptive response pattern, but this phenomenon is confirmed by microbiome science.

Microbiome science reflects this fundamental relationship between biological similars through the effects of different micro-organisms in the body. A wide diversity of commensal and symbiotic organisms are continuously necessary to promote cooperative ecological functioning, but certain potentially pathogenic organisms (pathobionts) are also critical for immune system regulation and developmental maturity. Pathobionts act synergistically in a balanced ecosystem and pathogenically in a dysbiotic system. These organisms are not only biologically important, but can also be critical in the regulation of immune function.

"A healthy, mature immune system depends on the constant intervention of beneficial bacteria. 'It goes against dogma to think that bacteria would make our immune systems function better, ... But the picture is getting very clear: the driving force behind the features of the immune system are commensals"'. 47

Acute infectious illness appears to be critical in immune system development. The greater the incidence of exposure to similar illnesses, the stronger the immune system's capacity to resist chronic inflammation. ${ }^{48}$ The immune system develops and learns by experience with actual infections, and the frequency of these infections is associated with reduced risk of developing allergic and inflammatory disorders. ${ }^{39}$ Young children with older brothers and sisters living at home, and those who attend a day-care center during the first 6 months of life, have a lower incidence of both asthma and type 1 diabetes than those who do not. Early life exposure to a wide range of infectious organisms reduces the risk of autoimmune conditions in later life. ${ }^{49}$ The risk of developing allergies is inversely linked with birth order and family size. The number of infections within households in early childhood is directly associated with a lower risk of developing allergic rhinitis (unless antibiotic treatment is used). ${ }^{50}$

Exposure to live infectious Mycobacterium species attenuates late-phase airway hyper-responsiveness and eosinophilia in mice with bronchial asthma. ${ }^{43}$ Early life pulmonary exposure to Mycobacteria protects against the development of allergic bronchial inflammation. ${ }^{51}$ The brains of mice exposed to live Mycobacteria prior to and during maze learning activities are associated with reduced anxiety-related behaviors and faster maze completion times when compared with placebo controls. ${ }^{32}$ A wide variety of pathobionts, including helminths, schistosomes and plasmodia species, demonstrate that exposure to living organisms capable of producing pathological changes in immunosuppressed individuals provides significant (paradoxical) benefit, preventing and treating a wide range of similar immune-related illnesses. ${ }^{52-59}$

\section{Individuality and Susceptibility}

Homeopathy works efficiently and effectively because it recognizes and utilizes differences in individuals, and treats them according to their varying susceptibility and sensitivity. No two individuals are ever exactly alike (and microbiome science confirms this); nor do they respond in precisely the same way to the same stimuli. Treatment that is individually tailored to match these differences is more likely to succeed.

Homeopathy depends on the science of identifying individual differences and selecting the most similar medicine to match them. If the goal of treatment is resolution of illness and inflammation, then this approach is critical and makes this outcome more likely. If the goal of treatment is to suppress symptoms as part of disease management (as in allopathic medicine), then individualization is unnecessary and has no bearing on outcome.

Many healing modalities recognize the benefits of individualization, including analytic psychology. According to Swiss psychoanalytic pioneer, Carl Gustav Jung:

"Analytical psychology, or Jungian psychology, emphasizes the primary importance of the individual psyche and the personal quest for wholeness." 60

Microbiome science confirms that each individual microbiome is unique and different, and that these differences are responsible for large variations in phenotypic gene expression and behavior. ${ }^{61}$ Differences between individual microbiomes account for many differences in the ways that individuals process and respond to stress. These differences vary considerably, and are tremendously reliable both clinically and forensically. ${ }^{62}$

Microbiome science confirms the phenomenon that in disease resolution and recovery of health, "one size does not fit all". Even from a purely biological perspective, remediation and replenishment of the microbiome through probiotics must be tailored to individual variation if it is to be effective. ${ }^{63}$ 
Most treatments tend to be more effective when they are aligned with individual differences: homeopathy proved this, while genetics and microbiome science confirm it.

\section{Minimum Dose}

The quantity of active material in doses of homeopathic medicines continues to be a source of conflict and controversy, ridicule, and skepticism; yet the phenomenon of delivering minuscule, infinitesimally small, quantities of homeopathic medicines continues to be supported by results both in pre-clinical studies and in placebo-controlled, double-blinded, clinical trials.

When all the criteria listed above are met-namely if: (1) treatment is individualized holistically to encompass the psychosomatic totality of an individual's characteristic and idiosyncratic symptoms; (2) the deepest levels of inflammation are addressed in accordance with the predicted direction of cure; and (3) the medicine is matched on the basis of the highest degree of similarity to the individual case at hand -then only minute, nanoscale amounts of single medicines, delivered in a few doses, are necessary to initiate homeostasis-inducing healing reactions throughout the body.

Materially large quantities (milligrams and grams) of drugs are typically necessary to initiate and sustain primary chemical drug actions in the body, particularly since these drugs (1) frequently depend on reaching high enough concentrations in the body to chemically saturate receptors on their target cells, and (2) are continuously subjected to active detoxification and elimination by the liver, kidneys, lungs, skin, and the GI tract. In the case of homeopathic medicines, delivered in nanoparticulate concentrations, working by secondary adaptive reactions, much smaller concentrations of medicine are necessary.

Homeopathic medicines can be viewed as working through the process known as hormesis ${ }^{27}$ (also known as the Arndt-Schulz rule or Schulz's law), which is a welldocumented, non-linear physiological and cellular adaption phenomenon characterized by a biphasic dose-response curve, demonstrating low-dose stimulation and high-dose inhibition effects. The phenomenon of hormesis matches homeopathic empiric evidence and demonstrates how all substances exhibit non-linear and biphasic dose-response curves (in opposite directions), as concentrations change. Convergent expert opinion suggests that hormesis is a nonlinear adaptive (not a pharmacological) process dependent on the reaction of a living organism, which utilizes it as a complex adaptive network response to stress. ${ }^{64}$

Microbiome and pharmaceutical sciences demonstrate that the phenomenon of hormesis can be utilized therapeutically (outside of homeopathy) to increase survival in patients with infectious diseases through the mechanism of improving disease tolerance, an adaptive defense mechanism that decreases the extent of infection-associated tissue damage without directly targeting pathogenic micro-organisms. Drugs known to target and inhibit host intracellular damage control and repair pathways (anthracyclines and other chemotherapeutic agents) are effective in treating infections and sepsis when used in nano-doses due to this hormetic phenomenon.

"At least theoretically, this [hormetic] approach should have the advantage as compared to antimicrobial agents in that development of resistance against the applied substances does not occur as no selection pressure is exerted on the pathogenic microorganisms."65

Homeopathic treatment demonstrates similar characteristics in infections, and it is logical to suspect that it works by the same mechanism. Substances that cause harm can be utilized (in nanoparticle or ultra-dilute "homeopathic" forms) to stimulate a return to homeostasis, and this is the basis of the Law of Similars. Patients treated adjunctively with homeopathy for severe sepsis in the intensive care unit demonstrate a non-statistically significant survival benefit at 30 days (verum homeopathy $81.8 \%$, placebo $67.7 \%, p=0.19$ ), and a statistically significant survival benefit on day 180 (verum homeopathy $75.8 \%$, placebo $50.0 \%, p=0.043$ ). ${ }^{66} \mathrm{Im}$ proved survival 6 months after acute treatment suggests that homeopathic treatment promotes an adaptive network response surpassing any direct chemical effects of treatment.

\section{Conclusion}

The discovery of the human microbiome marks one of the most critical junctures in medical history and an opportunity to shift the paradigm of medical thinking away from an ecologically destructive methodology toward one that promotes health and well-being. Microbiome science provides a unique opportunity to reflect on the science and practice of conventional and homeopathic medicine. The convergence of these two diametrically opposed fields is an "acid test" of microbiome science and ecology. Allopathic medicine demonstrates significant short- and long-term risks relating to all aspects of care, fails to improve long-term sustainable health, and causes significant damage to the microbiome, which results in an epidemic of chronic inflammatory illness and ecological devastation.

"It seems that the unhallowed principal business of the old school of medicine (allopathy) is to render incurable if not fatal the majority of diseases, those made chronic through ignorance by continually weakening and tormenting the already debilitated patient by the further addition of new destructive drug diseases." 67

On the other hand, homeopathic medicine finds both preclinical and clinical verification in microbiome science and exhibits significant benefit in short- and long-term efficacy, safety, and sustainability. It has been utilized worldwide for more than two centuries and has a track record documented by numerous studies and research investigations. These facts may be "inconvenient truths" since much of the world's (and $20 \%$ of the United States') economy is heavily vested in conventional medical education, research, and treatment.

Although homeopathy emerged from a vitalistic worldview, it is based on pure scientific empiricism, which has 
been qualitatively affirmed through molecular biology and one of the most brutally reductionistic, material investigations possible. Rather than rejecting or denying this science, it is critically important that it results in a shift of paradigm and a re-evaluation of assumptions. Funding should be diverted away from "status-quo" destructive and unsustainable treatment modalities toward research, teaching, and the clinical application of homeopathic methods. Countries such as India, which have already invested heavily in this infrastructure, should be emulated.

The world is faced with an environmental catastrophe of epic proportions in climate change, but the field of medicine faces a parallel epidemic of iatrogenic disability and chronic disease across all ages and socio-economic classes. The medical system that has been blithely followed has led to some destruction of health of individuals and of society. The impending global climate catastrophe and the medical destruction of our internal environments must be faced with the intelligent and the unbiased application of science (which, tragically, is not likely). Society must reject the "fake news" produced by organizations and associations that have vested interest in preventing change, and take action at all levels to shift health-care funding away from destructive modalities toward health-sustaining approaches.

The future of modern medicine and environmental health depends on grasping the meaning behind microbiome science and agreeing to work toward health-care and environmental ecology. Failure to take this emerging science seriously means failure to wrest control from business interests and a system capable of exploiting human and environmental health for mere profit.

\section{Highlights}

- Human health is intimately linked to the ecology and diversity of the human microbiome.

- Together, the human organism and the human microbiome form a complex super-organism.

- The principles of homeopathic medicine, including Holism, Psychosomatics, Direction of Cure, the Law of Similars, Individuality and Susceptibility, Minimum Dose, and Homeostasis, are all reflected in and substantiated by microbiome science.

- The only way forward in allopathic medicine is to abandon unsustainable practices that damage the microbiome and promote chronic illness.

- The future of medicine depends on incorporating microbiome science, and one of the most practical ways of doing this is through homeopathy.

\section{Conflict of Interest}

None declared.

\section{References}

1 Gorman J. Aliens Inside Us: A (Mostly Friendly) Bacterial Nation. New York Times; 2003:F3

2 Bashiardes S, Zilberman-Schapira G, Elinav E. Use of metatranscriptomics in microbiome research. Bioinform Biol Insights 2016;10:19-25
3 Servick K. Do gut bacteria make a second home in our brains? Science 2018November9

4 Adolph TE, Grander C, Moschen AR, Tilg H. Liver-microbiome axis in health and disease. Trends Immunol 2018;39:712-723

5 Aagaard K, Ma J, Antony KM, Ganu R, Petrosino J, Versalovic J. The placenta harbors a unique microbiome. Sci Transl Med 2014;21;6:237ra65

6 Baker JM, Chase DM, Herbst-Kralovetz MM. Uterine microbiota: residents, tourists, or invaders? Front Immunol 2018;9:208

7 Païssé S, Valle C, Servant F, et al. Comprehensive description of blood microbiome from healthy donors assessed by $16 \mathrm{~S}$ targeted metagenomic sequencing. Transfusion 2016;56:1138-1147

8 Kwon D. The connection was electric. Sci Am 2016;314:20

9 Simard SW. Mycorrhizal networks facilitate tree communication, learning and memory. In: Baluska F, Gagliano M, Witzany G, eds. Memory and Learning in Plants. Chap. 10. Springer; 2018:191-213

10 Li K, Bihan M, Yooseph S, Methé BA. Analyses of the microbial diversity across the human microbiome. PLoS One 2012;7:e32118

11 Sleator RD. The human superorganism - of microbes and men. Med Hypotheses 2010;74:214-215

12 Turnbaugh PJ, Ley RE, Hamady M, Fraser-Liggett CM, Knight R, Gordon JI. The human microbiome project. Nature 2007; 449:804-810

13 Pastuzyn ED, Day CE, Kearns RB, et al. The neuronal gene arc encodes a repurposed retrotransposon gag protein that mediates intercellular RNA transfer. Cell 2018;172:275-288.e18

14 Milani RV, Lavie CJ. Health care 2020: reengineering health care delivery to combat chronic disease. Am J Med 2015;128:337-343

15 Nguyen LDN, Viscogliosi E, Delhaes L. The lung mycobiome: an emerging field of the human respiratory microbiome. Front Microbiol 2015;6:89

16 Gritz EC, Bhandari V. The human neonatal gut microbiome: a brief review. Front Pediatr 2015;3:17

17 O'Hara AM, Shanahan F. The gut flora as a forgotten organ. EMBO Rep 2006;7:688-693

18 Sampson TR, Mazmanian SK. Control of brain development, function, and behavior by the microbiome. Cell Host Microbe 2015;17:565-576

19 Aroniadis OC, Brandt LJ. Fecal microbiota transplantation: past, present and future. Curr Opin Gastroenterol 2013;29:79-84

20 Bhattacharjee S, Lukiw WJ. Alzheimer's disease and the microbiome. Front Cell Neurosci 2013;7:153

21 Berrington JE, Stewart CJ, Embleton ND, Cummings SP. Gut microbiota in preterm infants: assessment and relevance to health and disease. Arch Dis Child Fetal Neonatal Ed 2013;98:F286-F290

22 Hahnemann S. Organon of the Medical Art. Aphorism 9. In: O'Reilly WB, ed. Redmond, WA: Birdcage Books; 1996:65

23 Paterson J. The potentised drug and its action on the bowel flora. Br Homeopath J 1936;26:163-188

24 Saha SK, Das S, Khuda-Bukhsh AR. Phenotypic evidence of ultrahighly diluted homeopathic remedies acting at gene expression level: a novel probe on experimental phage infectivity in bacteria. J Chin Integr Med 2012;10:462-470

25 Nelson LS, Juurlink DN, Perrone J. Addressing the opioid epidemic. JAMA 2015;314:1453-1454

26 Centers for Disease Control and Prevention. Antibiotic use in outpatient settings, 2017. Available at: https://www.cdc.gov/ antibiotic-use/stewardship-report/outpatient.html

27 Bell IR. The complexity of the homeopathic healing response Part 1: The role of the body as a complex adaptive system in simillimum-initiated recovery from disease. Homeopathy 2020; 109:42-50

28 Lazarou J, Pomeranz BH, Corey PN. Incidence of adverse drug reactions in hospitalized patients: a meta-analysis of prospective studies. JAMA 1998;279:1200-1205

29 Prescribers' Digital Reference. Available at: https://www.pdr.net/

30 Cryan JF, O'Mahony SM. The microbiome-gut-brain axis: from bowel to behavior. Neurogastroenterol Motil 2011;23:187-192 
31 Grenham S, Clarke G, Cryan JF, Dinan TG. Brain-gut-microbe communication in health and disease. Front Physiol 2011;2:94

32 Matthews DM, Jenks SM. Ingestion of Mycobacterium vaccae decreases anxiety-related behavior and improves learning in mice. Behav Processes 2013;96:27-35

33 Roberts RC, Farmer CB, Walker CK. The human brain microbiome; there are bacteria in our brains! Psych Behav Neurobio 2018. U Alabama, Birmingham, Birmingham, AL. Program No. 594.08. 2018 Neuroscience Meeting Planner. San Diego, CA: Society for Neuroscience, Online

34 Valasquez-Manoff M. An Epidemic of Absence. Scribner, NY2012

35 WholeHealthNow. Dr. Constantine Hering M.D. (1800-1880). Available at: http://www.wholehealthnow.com/bios/constantine-hering.html

36 Vijayakar P. Predictive Homeopathy. Mumbai, India: Shree Smaran Enterprises; 2003

37 Kivellos S, Skifti S, Vithoulkas G. EHMTI-0396. Reappearance of high fever on migraine patients, after individualized homeopathic treatment, is a valuable prognostic factor. J Headache Pain 2014; 15(Suppl 1):M7

38 Kozyrskyj AL, Ernst P, Becker AB. Increased risk of childhood asthma from antibiotic use in early life. Chest 2007; 131:1753-1759

39 Wickens K, Pearce N, Crane J, Beasley R. Antibiotic use in early childhood and the development of asthma. Clin Exp Allergy 1999; 29:766-771

40 Foliaki S, Pearce N, Björkstén B, Mallol J, Montefort S, von Mutius E; International Study of Asthma and Allergies in Childhood Phase III Study Group. Antibiotic use in infancy and symptoms of asthma, rhinoconjunctivitis, and eczema in children 6 and 7 years old: international study of asthma and allergies in childhood phase III. J Allergy Clin Immunol 2009;124:982-989

41 Droste JH, Wieringa MH, Weyler JJ, Nelen VJ, Vermeire PA, Van Bever HP. Does the use of antibiotics in early childhood increase the risk of asthma and allergic disease? Clin Exp Allergy 2000; 30:1547-1553

42 Dickson RP, Martinez FJ, Huffnagle GB. The role of the microbiome in exacerbations of chronic lung diseases. Lancet 2014; 384:691-702

43 Bach JF. The effect of infections on susceptibility to autoimmune and allergic diseases. N Engl J Med 2002;347:911-920

44 Mahesh S, Jaggi L, Jaggi A, Tsintzas D, Vithoulkas G. Individualised homeopathic therapy in ANCA negative rapidly progressive necrotising crescentic glomerulonephritis with severe renal insufficiency - a case report. J Med Life 2019;12:49-55

45 Doux JD, Bazar KA, Lee PY, Yun AJ. Can chronic use of antiinflammatory agents paradoxically promote chronic inflammation through compensatory host response? Med Hypotheses 2005;65:389-391

46 Yun AJ, Lee PY, Bazar KA. Paradoxical strategy for treating chronic diseases where the therapeutic effect is derived from compensatory response rather than drug effect. Med Hypotheses 2005; 64:1050-1059

47 Ackerman J. The ultimate social network. Sci Am 2012;306:36-43

48 Ball TM, Castro-Rodriguez JA, Griffith KA, Holberg CJ, Martinez FD, Wright AL. Siblings, day-care attendance, and the risk of asthma and wheezing during childhood. N Engl J Med 2000; 343:538-543

49 McKinney PA, Okasha M, Parslow RC, et al. Early social mixing and childhood Type 1 diabetes mellitus: a case-control study in Yorkshire, UK. Diabet Med 2000;17:236-242

50 Strachan DP. Hay fever, hygiene, and household size. BMJ 1989; 299:1259-1260

51 Zuany-Amorim C, Sawicka E, Manlius C, et al. Suppression of airway eosinophilia by killed Mycobacterium vaccae-induced allergen-specific regulatory T-cells. Nat Med 2002;8:625-629

52 Vermund $\mathrm{SH}$, MacLeod S. Is pinworm a vanishing infection? Laboratory surveillance in a New York City medical center from 1971 to 1986 . Am J Dis Child 1988;142:566-568

53 Cooper PJ, Barreto ML, Rodrigues LC. Human allergy and geohelminth infections: a review of the literature and a proposed conceptual model to guide the investigation of possible causal associations. Br Med Bull 2006;79-80:203-218

54 Lynch NR, Hagel I, Perez M, Di Prisco MC, Lopez R, Alvarez N. Effect of anthelmintic treatment on the allergic reactivity of children in a tropical slum. J Allergy Clin Immunol 1993;92:404-411

55 Liu J, Morey RA, Wilson JK, Parker W. Practices and outcomes of self-treatment with helminths based on physicians' observations. J Helminthol 2017;91:267-277

56 Khan WI, Blennerhasset PA, Varghese AK, et al. Intestinal nematode infection ameliorates experimental colitis in mice. Infect Immun 2002;70:5931-5937

57 Elliott DE, Li J, Blum A, et al. Exposure to schistosome eggs protects mice from TNBS-induced colitis. Am J Physiol Gastrointest Liver Physiol 2003;284:G385-G391

58 Leonardi-Bee J, Pritchard D, Britton J. Asthma and current intestinal parasite infection: systematic review and meta-analysis. Am J Respir Crit Care Med 2006;174:514-523

59 Cheng AM, Jaint D, Thomas S, Wilson JK, Parker W. Overcoming evolutionary mismatch by self-treatment with helminths: current practices and experience. J Evolutionary Med 2015;3:235910

60 Journal Psyche. Jung and his individuation process. Available at: http://journalpsyche.org/jung-and-his-individuation-process/

61 Eckburg PB, Bik EM, Bernstein CN, et al. Diversity of the human intestinal microbial flora. Science 2005;308:1635-1638

62 National Institute of Justice. Forensic applications of microbiomes. Available athttps://nij.ojp.gov/topics/articles/forensicapplications-microbiomes

63 Rao SSC, Rehman A, Yu S, Andino NM. Brain fogginess, gas and bloating: a link between SIBO, probiotics and metabolic acidosis. Clin Transl Gastroenterol 2018;9:162

64 Bell IR, Schwartz GE, Boyer NN, Koithan M, Brooks AJ. Advances in integrative nanomedicine for improving infectious disease treatment in public health. Eur J Integr Med 2013;5:126-140

65 Weis S, Rubio I, Ludwig K, Weigel C, Jentho E. Hormesis and defense of infectious disease. Int J Mol Sci 2017;18:1273

66 Frass M, Linkesch M, Banyai S, et al. Adjunctive homeopathic treatment in patients with severe sepsis: a randomized, doubleblind, placebo-controlled trial in an intensive care unit. Homeopathy 2005;94:75-80

67 Hahnemann S. Organon of the Medical Art. 6th ed. New Delhi, India: Jain Publishers; 17 